\title{
Carvedilol in Patients with Acutely Decompensated Systolic Heart Failure: Effects on Survival
}

\author{
Francisco Jose Sánchez Rivas ${ }^{1,2}$, Jose Hipolito Donis Hernández ${ }^{1, *}$, Carmen Mazzei De Dávila ${ }^{3}$, \\ Maite Alexandra González ${ }^{1}$, Diego Fernando Dávila ${ }^{1,2}$ \\ ${ }^{1}$ Faculty of Medicine, Department Medicine, Cardiovascular Investigation Institute, The Andes University Hospital, The Andes University, \\ Mérida, Venezuela \\ ${ }^{2}$ Social Security Institute, Mérida, Venezuela \\ ${ }^{3}$ Department de Paediatrics, The Andes University Hospital, Mérida, Venezuela
}

\section{Email Address:}

donis_jose@hotmail.com (J. H. D. Hernández)

${ }^{*}$ Corresponding author

\section{To cite this article:}

Francisco Jose Sánchez Rivas, Jose Hipolito Donis Hernández, Carmen Mazzei De Dávila, Maite Alexandra González, Diego Fernando Dávila. Carvedilol in Patients with Acutely Decompensated Systolic Heart Failure: Effects on Survival. American Journal of Internal Medicine. Vol. 9, No. 4, 2021, pp. 186-193. doi: 10.11648/j.ajim.20210904.14

Received: June 19, 2021; Accepted: July 5, 2021; Published: July 9, 2021

\begin{abstract}
Ninety-eight patients with acutely decompensated systolic heart failure were admitted to the Hospital Universitario de Los Andes between 2005 and 2011, in Mérida, Venezuela. Medical Treatment: Protocol 1: Furosemide 20 mg IV every 8 hours (28 patients). Protocol 2: Furosemide 20 IV every 24 hours plus cautious uptitration of carvedilol (70 patients). Heart rate decreased from $99.19 \pm 12.38$ to $67.64 \pm 11.27(\mathrm{bpm})(\mathrm{p}<0.0001)$ with protocol 2. Daily weight changes were similar both protocols. Mean maximum dose of carvedilol was $59.37 \mathrm{mg}$, furosemide $240 \mathrm{mg}$ for protocol 1 and $80 \mathrm{mg}$ for protocol 2. For the whole group of patients, survival probability was close to $60 \%$ at fifty months of follow up. There were fourteen deaths with protocol 1 and eleven with protocol 2. Survival probability was significantly higher, in patients assigned to protocol 2 versus protocol 1 ( $72 \%$ vs $38 \%, p<0.046)$. Cox multiple regression analysis indicated that, medical treatment with carvedilol, was significantly and independently associated to survival, only in those patients who were in sinus rhythm. Cautious uptitration of carvedilol, in still decompensated patients with sinus rhythm, increases long term survival.
\end{abstract}

Keywords: Furosemide, Carvedilol, Acute Decompensated Systolic Heart Failure, Heart Rate, Survival Probability

\section{Introduction}

The natural history of patients with chronic systolic heart failure is characterized by the recurrence of congestive signs and symptoms [1] These episodes, of acutely decompensated heart failure, appear within one hundred days to six months post-discharge and are associated to diminished survival [2,3]. The onset of decompensation is usually gradual, fluid overload predominates over decreased tissue perfusion [4-8] and there is biochemical evidence of neurohormonal activation [9, 10] and myocytolisis [11]. The results of current therapeutic strategies, based on frequent and high doses of diuretics, increase morbidity and mortality [12-15]. Although, its use is still controversial, in hypervolemic uncompensated patients $[16,17]$, a cardioprotective strategy with beta-adrenergic blockers appears to improve survival $[18,19]$. Beta blockers are contraindicated in patients with acutely decompensated heart failure. Current therapeutic strategies increase morbidity and mortality. We have compared the effects of frequent doses of diuretics vs a single dose of diuretics and cautious uptitration of carvedilol. Our results indicate that, although clinical compensation is achieved with both strategies; the effects on neurohormonal activation and ventricular arrhythmias are opposite and we previously reported the short-term effects of these two opposite strategies, Consequently, selected patients with acutely decompensated heart failure can be compensated, during a $96 \mathrm{~h}$ period of observation, with a cautious uptitration of carvedilol and single daily dose of diuretics [20]. We previously reported the short term effects of these two opposite strategies and now 
describe their long term effects on survival.

\section{Methods}

We retrospectively reviewed the medical records of patients admitted, with acutely decompensated heart failure, to the Hospital Universitario de Los Andes and to the Instituto Venezolano del Seguro Social in Mérida, Venezuela, between 2005 and 2011. All patients were congestive, normothermic and with adequate perfusion pressure (Systolic blood pressure $>$ $90 \mathrm{mmHg}$ (Profile B, Functional class III/IV) [7]. Medical treatment was based on two opposite therapeutic strategies [20]. Protocol 1: Furosemide $20 \mathrm{mg}$ IV every 8 hours and Protocol 2: Furosemide $20 \mathrm{mg}$ IV every 24 hours plus cautious up titration of carvedilol. Uptitration of carvedilol was carried out by increasing the initial dose of $3.125 \mathrm{mg}$, by $3.125 \mathrm{mg}$ every 12 hours. Uptitration was heart rate oriented (Target: 65-70 bpm) and preceded by a thorough clinical evaluation. Betablockers on admission were switched to carvedilol [Protocol 2]. Patients in both protocols received digitalis and prophylaxis for deep venous thrombo-embolism. Captopril $6.25 \mathrm{mg}$ every 8 hours was also administrated to protocol 1 patients. Two-dimensional transthoracic echocardiogram was performed upon admission and daily dry weight was determined every 24 hours, during the observation period of 96 hours. Upon termination of the in-hospital observation period, patients were discharged and followed in the outpatient clinic. Standard treatment for chronic congestive heart failure was now administered to patients in both protocols $[16,17]$. Protocols had been previously approved by the Commission for Clinical Research of the Instituto de Investigations Cardiovascular of the University of Los Andes. Informed consent was obtained from all participants in the study.

\section{Statistical Analysis}

Data are expressed as absolute numbers and percentages. The one sample Kolmogorov-Smirnov and the Shapiro-Wilk tests were used to analyze for normal or not normal distribution of the data. Continuous normally distributed variables are expressed as mean \pm standard deviation. Intra e intergroup comparisons for daily heart rate and dry weight changes were performed by means of repetitive analysis of variance. Survival probability was estimated by the Kaplan-Meier method and differences in survival between groups were assessed by the log-rank test. Cox regression analysis was used to determine a possible association between survival and potentially explanatory independent variables such as: Medical treatment, age, heart rhythm and the absolute changes in heart rate and daily weight. Statistical significance was considered for $\mathrm{p}<0.05$.

\section{Results}

Baseline demographic, clinical and echocardiographic characteristics. The medical records of ninety-eight patients were identified. Initially, patients were consecutively assigned to each protocol [Protocol 1: 21 patients and Protocol 2: 23]. However, during the last four years [20072011] most patients received protocol 2 [47 patients] and the remainder Protocol 1 [7 patients]. Baseline characteristics for all patients are shown in Table 1. Mean age was $64.87 \pm 13.04$ years and males predominated. Baseline heart rate was $97.41 \pm 14.73$ beats per minute and systolic blood pressure $129.44 \pm 17.84 \mathrm{mmHg}$. Most patients were in functional class III [NYHA 58\%]. Sinus rhythm was present in more than half [52.24\%] and atrial fibrillation in the remaining patients [47.76\%]. Renal function was borderline and the most frequently prescribed drug was furosemide. All patients had severely depressed left ventricular function [Ejection fraction: [28.61 \pm 13.54$]$ and increased pulmonary wedge pressure $[28.61 \pm 13.54 \mathrm{~mm} \mathrm{Hg}]$ [21]. As can be seen in Table 2, patients assigned to protocol 2 had higher baseline heart rate, diastolic blood pressure, serum creatinine and left ventricular ejection fraction. Mean maximum dose of carvedilol for the 96 hours observation period was $59.37 \mathrm{mg}$, furosemide $240 \mathrm{mg}$ for protocol 1 and $80 \mathrm{mg}$ for protocol 2 and captopril $75 \mathrm{mg}$.

Table 1. Baseline demographic, clinical and echocardiographic characteristics.

\begin{tabular}{ll}
\hline Characteristics & Median. $(\mathbf{n}=\mathbf{9 8})$ \\
\hline Demographic variables. & \\
Age (years) & $64.87 \pm 13.04$ \\
Sex (M/F) & $79 / 19$ \\
Clinical variables. & $70.46 \pm 12.45$ \\
Weight (kg) & $97.41 \pm 14.73$ \\
Heart rate (beats per minute) & $129.44 \pm 17.84$ \\
Systolic pressure (mmHg) & $83.97 \pm 11.67$ \\
Diastolic pressure (mmHg) & $69 / 29$ \\
NYHA Functional class (III/IV) & $69 / 29$ \\
Heart rhythm (Sinus rhythm/Atrial Fibrillation) & \\
Aetiology (\%) & 25.64 \\
Hypertensive & 15.38 \\
Ischemic & 38.47 \\
Mixed & 2.56 \\
Chagásic & \\
\hline
\end{tabular}




\begin{tabular}{ll}
\hline Characteristics & Median. $\mathbf{( n = 9 8 )}$ \\
\hline Idiopathic & 17.95 \\
Treatment (n / \%) & $98 / 100$ \\
Furosemide & $45 / 44.1$ \\
Digital & $29 / 28.42$ \\
Captopril & $60 / 58.8$ \\
Beta-blockers & \\
Laboratory & $1.54 \pm 0.57$ \\
Creatine (mg/dl) & $4.13 \pm 0.51$ \\
Potassium (mEq/lt) & $6.55 \pm 0.48$ \\
Proteins (g/\%) & \\
Echocardiography. & $25.18 \pm 8.82$ \\
Ejection fraction (\%) & $0.73 \pm 0.07$ \\
Sphericity Index & $61.15 \pm 5.62$ \\
Left ventricular diastolic diameter $(\mathrm{mm})$ & $188.12 \pm 40.97$ \\
Left ventricular diastolic volume $(\mathrm{cc})$ & $20.97 \pm 10.01$ \\
Relation E/e' & $28.61 \pm 13.54$ \\
Wedge Pressure for (mmHg) & \\
\hline
\end{tabular}

Table 2. Demographic and Baseline Characteristics of the Study Patients for Group.

\begin{tabular}{|c|c|c|c|}
\hline Characteristics & Group n: 28 & Group n: 70 & P Value \\
\hline \multicolumn{4}{|l|}{ Demographic variables. } \\
\hline Age (yrs) & $63.04 \pm 13.04$ & $65.60 \pm 12.46$ & \\
\hline Sex. $(M / F)$ & $21 / 7$ & $58 / 12$ & \\
\hline \multicolumn{4}{|l|}{ Clinical variables. } \\
\hline Weight $(\mathrm{kg})$ & $66,32 \pm 11.6$ & $72.35 \pm 12.87$ & \\
\hline Heart rate $(\mathrm{bpm}) £$ & $91.37 \pm 14,92$ & $99.19 \pm 12.38$ & 0.03 \\
\hline Systolic pressure (mmHg) & $119 \pm 19,67$ & $133.61 \pm 15.31$ & \\
\hline Diastolic pressure $(\mathrm{mmHg}) £$ & $79.11 \pm 13.16$ & $85.91 \pm 10.50$ & 0.04 \\
\hline (NYHA) Functional class (III/IV) & $19 / 9$ & $50 / 20$ & \\
\hline Heart Rhythm (RS/AF) & $19 / 9$ & $50 / 20$ & \\
\hline \multicolumn{4}{|l|}{ Etiology $(\%)$} \\
\hline Hypertensive & 35.71 & 38.57 & \\
\hline ischemic & 21.43 & 41.43 & \\
\hline Mixed & 21.43 & 17.14 & \\
\hline Chagásica & 3.57 & 0 & \\
\hline Idiopathic & 17.86 & 2.86 & \\
\hline \multicolumn{4}{|l|}{ Treatment of revenue $(\mathrm{n} \% \%)$} \\
\hline Furosemide & $28 / 100$ & $70 / 100$ & \\
\hline Digital & $15 / 53.57$ & $30 / 42.85$ & \\
\hline Captopril & $20 / 71.43$ & $9 / 12.86$ & \\
\hline Beta-blocking & $15 / 53.57$ & $45 / 64.28$ & \\
\hline \multicolumn{4}{|l|}{ Laboratory } \\
\hline Creatine $(\mathrm{mg} / \mathrm{dl}) £$ & $1,45 \pm 0.31$ & $1,65 \pm 0.76$ & 0.08 \\
\hline Potassium $(\mathrm{mEq} / \mathrm{lt})$ & $3.96 \pm 0.25$ & $4.36 \pm 0.68$ & \\
\hline Proteins $(\mathrm{g} / \mathrm{dl})$ & $6.44 \pm 0.47$ & $6.72 \pm 0.49$ & \\
\hline \multicolumn{4}{|l|}{ Echocardiography. } \\
\hline Ejection fraction FEVI (\%) $£$ & $20.10 \pm 5.60$ & $26,85 \pm 9.08$ & 0.008 \\
\hline Esfericidad Of Index & $0.74 \pm 0.07$ & $0,74 \pm 0.7$ & \\
\hline LVDD $(\mathrm{mm})$ & $60.59 \pm 5.16$ & $59.57 \pm 6.47$ & \\
\hline Relation E/e' & $21.87 \pm 11,28$ & $20,00 \pm 8,68$ & \\
\hline Wedge of Pressure for Nagueh $(\mathrm{mmHg})$ & $30.55 \pm 16, .0$ & $26, .5 \pm 9.00$ & \\
\hline
\end{tabular}

\section{Effects on Clinical Variables and Mortality}

Heart rate decreased from $99.19 \pm 12.38$ bpm to $67.64 \pm 11.27$ $[\mathrm{p}<0.0001]$, in protocol 2 patients but, it remained unchanged in protocol 1 patients [Figure 1]. Daily dry weight decreased significantly, in both groups of patients, during the four days observation period. Intergroup comparisons for the absolute daily changes in dry weight were similar [Figure 2]. Daily dry weight decreased significantly, in both groups of patients, during the four days observation period. Intergroup comparisons for the absolute daily changes in dry weight were similar [Figure 2]. For the whole group of patients, survival probability was close to $60 \%$ at fifty months of follow up [Figure 3].

For the whole group of patients, survival probability was close to $60 \%$ at fifty months of follow up [Figure 3]. 


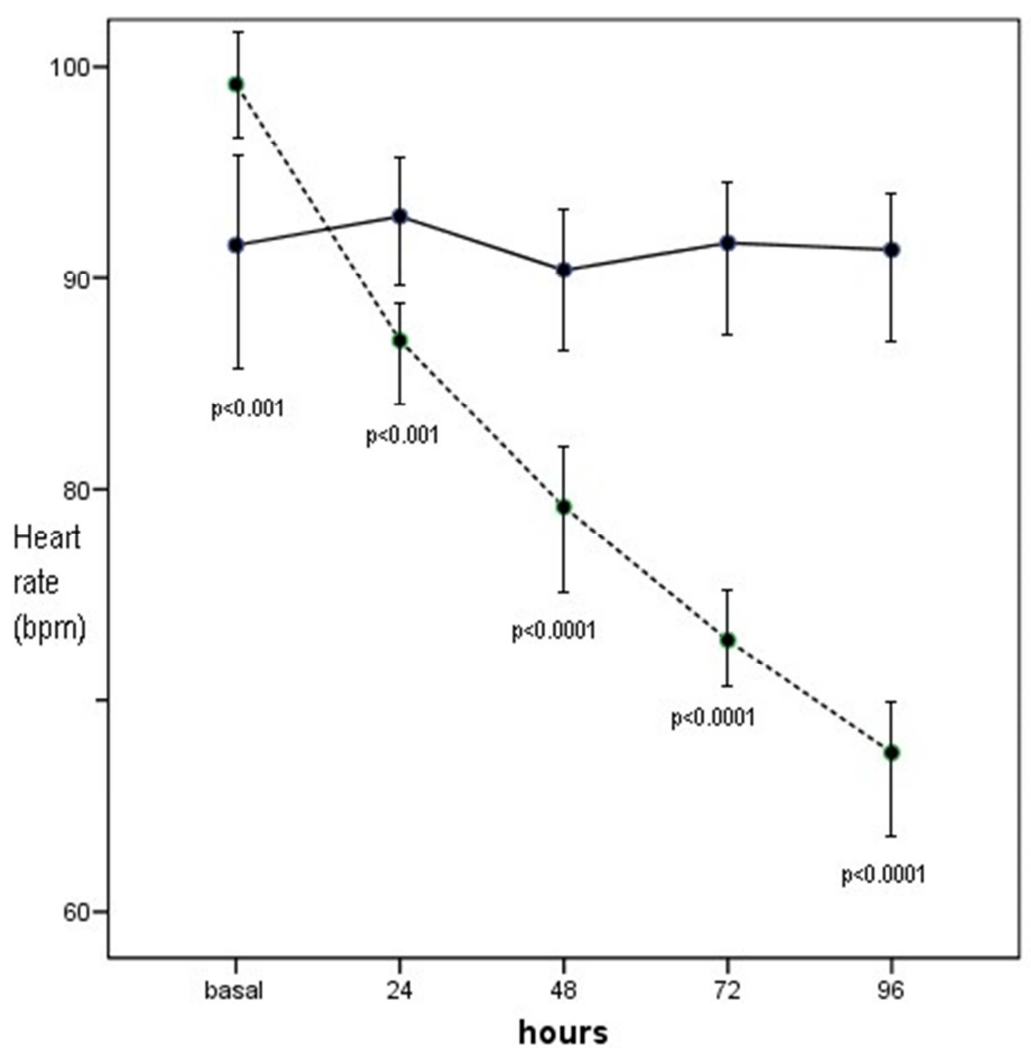

- Protocol 1

.....- Protocol 2

Figure 1. Heart rate changes with furosemide (Protocol 1) versus carvedilol (Protocol 2). Heart rate decreased significantly with carvedilol, but it remained unchanged with furosemide.

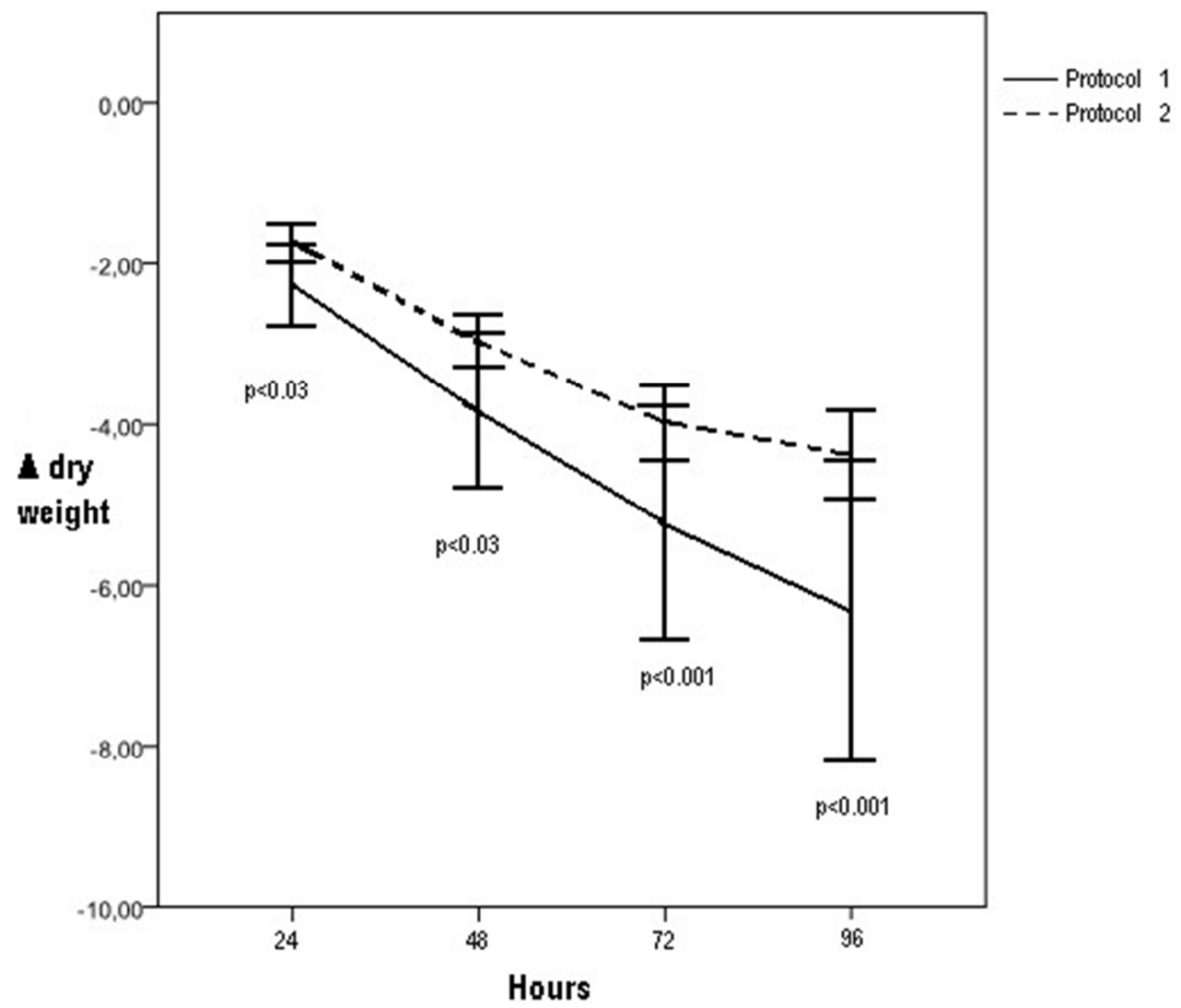

Figure 2. Absolute daily changes in dry weight with furosemide (Protocol 1) versus Carvedilol (Protocol 2). Weight decreased significantly, during the 96 hours observation period, with both protocols. However, intergroup comparisons revealed no significant differences. $\boldsymbol{\Delta}=$ Absolute changes in dry weight. 


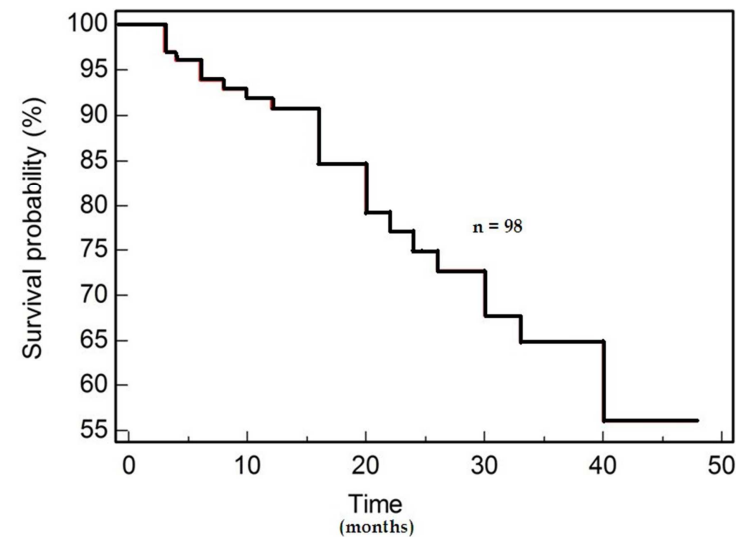

Figure 3. Survival probability for the entire group of patients ( $n=98)$. For the whole group of patients, survival probability was close to $60 \%$, at fifty months of follow up.

There were fourteen deaths with protocol 1 and eleven with protocol 2. According to use or not use of carvedilol, survival probability was significantly higher, in patients assigned to protocol 2 versus protocol 1 [72\% vs 38\%, p< 0.046] [Figure 4]. Discrimination of patients in sinus rhythm versus atrial fibrillation showed a higher survival only in the former (Figures 5 and 6). The magnitude of the heart rate change, with carvedilol in patients in sinus rhythm or in atrial fibrillation, was not statistically different [Figure 7]. Cox multiple regression analysis indicated that, medical treatment with carvedilol, was significantly and independently associated to survival, only in those patients who were in sinus rhythm [Tables 3 and 4].

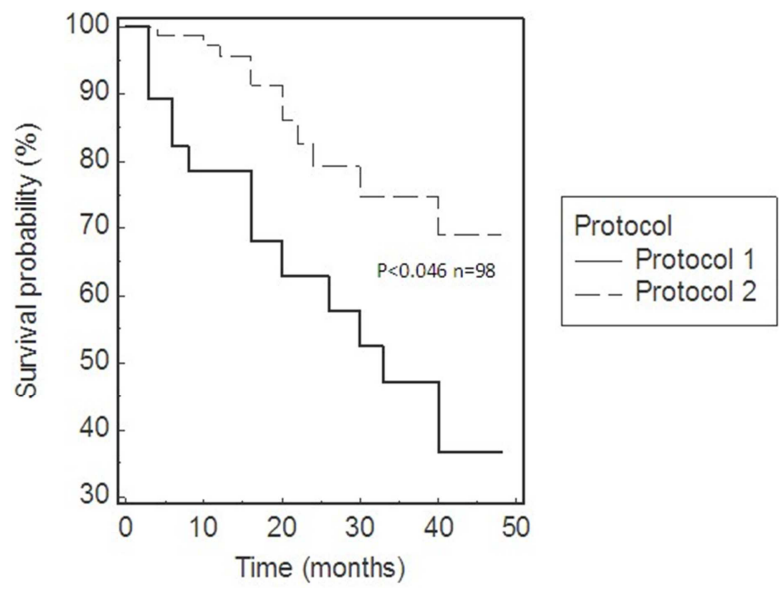

Figure 4. Survival probability for decompensated patients in sinus rhythm and in atrial fibrillation. Survival was significantly lower with Protocol 1 (Furosemide) versus Protocol 2 (Carvedilol).

Table 3. Multivariate analysis by Cox regression model for patients in sinus rthmths.

\begin{tabular}{llll}
\hline Variable Basal & $\boldsymbol{\beta}$ Coefficient & Hazard Ratio (95\% CI) & P Value \\
\hline Medical treatment & -1.171 & $0.310(0.101$ to 0.954$)$ & 0.041 \\
Age & 0.025 & $2.070(0.991$ to 1.061$)$ & 0.150 \\
$\Delta$ Weigth & 0.083 & $1.086(0.900$ to 1.311$)$ & 0.389 \\
$\Delta$ Heart rate & 0.027 & $1.027(0.9623$ to 1.095$)$ & 0.415 \\
\hline
\end{tabular}

$\Delta$ Absolute changes

Table 4. Multivariate analysis by Cox regression model for patients in atrial fibrillation.

\begin{tabular}{llll}
\hline Variable Basal & $\boldsymbol{\beta}$ Coefficient & Hazard Ratio (95\% CI) & Palue \\
\hline Medical treatment & -0.948 & $0.388(0.084$ to 1.780$)$ & 0.223 \\
Age & 0.025 & $1.025(0.959$ to 1.096$)$ & 0.469 \\
$\Delta$ Weight & 0.066 & $0.936(0.832$ to 1.053$)$ \\
$\Delta$ Heart rate & 0.074 & $1.077(0.987$ to 1.176$)$ & 0.271 \\
\hline
\end{tabular}

$\Delta$ Absolute Chages

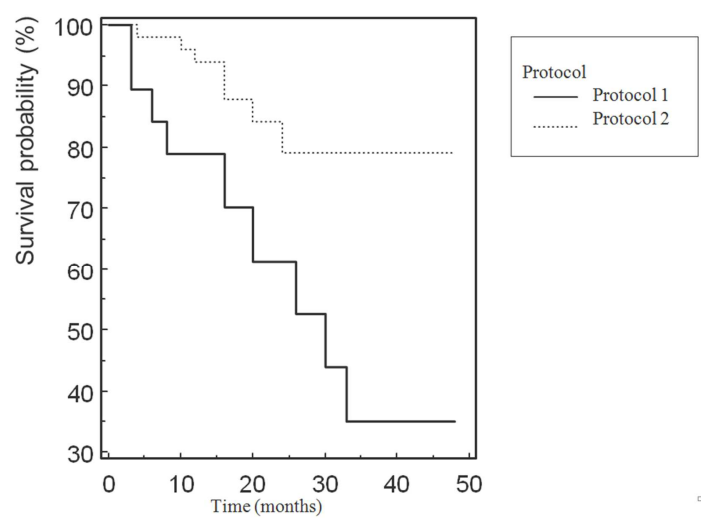

Figure 5. Survival probability for decompensated patients in sinus rhythm. Survival was significantly lower with Protocol 1 (Furosemide) versus Protocol 2 (Carvedilol).

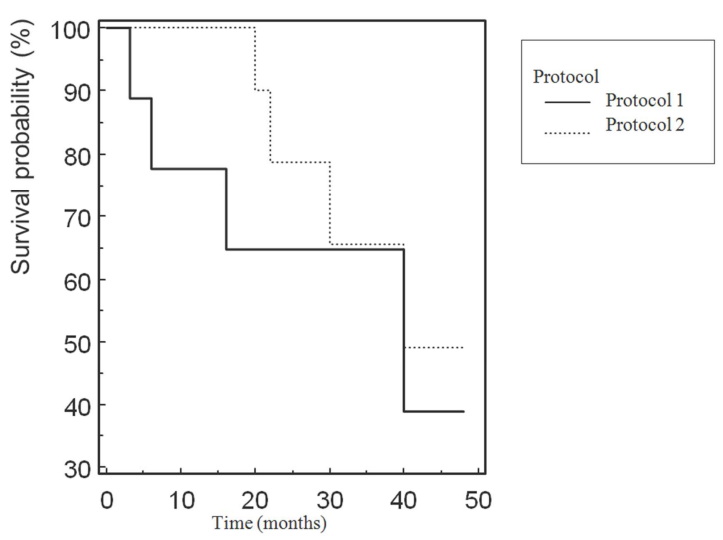

Figure 6. Survival probability for decompensated patients in atrial fibrilation. Survival was not significantly different with Protocol 1 (Furosemide) versus Protocol 2 (Carvedilol). 


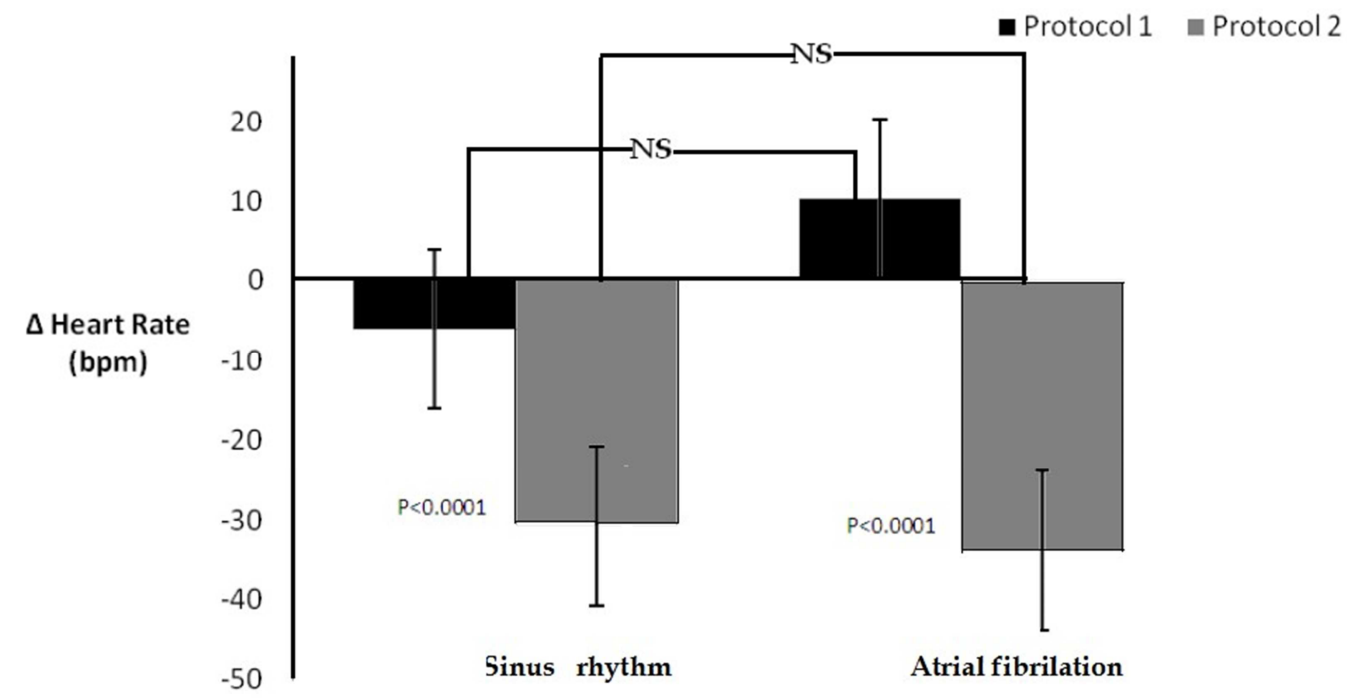

Figure 7. Magnitude of the heart rate changes in patients in sinus rhythm or in atrial fibrillation. Heart rate decreased markedly and significantly in both groups of patients with Protocol 2 (Carvedilol), vs Protocol 1 (Furosemide). The magnitude of these negative heart rate changes were similar in patients with sinus rhythm compared to patients with atrial fibrillation.

\section{Discussion}

The pathophysiology of acute decompensation, of chronic and stable heart failure patients, is still incompletely understood. Possible mechanisms are non-adherence to diet or pharmacological therapy, arrhythmias, impaired cardiac contractility, and renal insufficiency. All of these abnormalities lead to or contribute with neurohormonal activation, progressive fluid retention, body weight gain and congestion [22]. More recently, Fallick C et al., proposed that, a sympathetically mediated shift between extracellular fluid volumen and effective circulating blood volumen, would partially explain the development of congestion, even in the absence of weight gain [23]. Since alpha receptors predominate in the splanchnic blood reservoir [24], those investigators went on to state that: "Although, $\beta$ blockade is still contraindicated in the setting of acute decompensation, perhaps judicious use of combined $A$ and $\beta$ blockade could be considered in the future".

For the past seven years, we at the Instituto de Investigations Cardiovascular of the University of Los Andes in Mérida, Venezuela have been compensating our systolic heart failure patients with carvedilol [20]. The rationality, for comparing this therapeutic strategy versus the conventional use of high and frequent doses of furosemide, was as follows: 1. Acute decompensation is characterized by congestion, neurohormonal activation and myocytolisis [4-11]. 2 . Furosemide enhances neurohormonal activation [25] and increases morbidity and mortality [12-15]. 3. The first report on the beneficial effects of the non-selective beta blocker practolol, published in 1975, included patients who were still hypervolemic [26]. The U.S. Carvedilol Heart Failure Study Group also included hypervolemic patients as a high risk group. These still uncompensated patients had a long-term survival similar to that of euvolemic patients [27].
The Kaplan-Mier analysis of our database showed that, for the whole group of patients, survival probability was close to $60 \%$ at fifty months of follow up. However, patients receiving carvedilol, had a better survival than those assigned to high and frequent dosis of furosemide. Patients in sinus rhythm, compared to those with atrial fibrillation as the predominate heart rhythm, were the only ones to have an increased survival. Cox regression analysis confirmed that, carvedilol and sinus rhythm, were the only variables independently associated with survival [Tables 3 and 4]. Recent prospective and retrospective studies, in decompensated patients, have paid particular attention to the relationship of continuation, withdrawal or newly starting of beta blockers [18, 19, 28, 29]. All of these studies consistently demonstrated that, short term cardiac mortality and morbidity, were significantly lower in those patients newly started or continued on beta blockers. Our findings indicate that long term survival is also positively influenced by the administration of carvedilol, to acutely decompensated patients.

Why is the non-selective beta blocker carvedilol tolerated by decompensated patients and at the same time associated with increased survival? First of all, we should emphasize that, our patients were B category of the classification proposed by Nohria A., et al [7]. They were predominantly congestive, with adequate perfusion pressure and their baseline heart rate decreased gradually over the 96 hours observation period. Thus, cardiac sympathetic drive and its well-known deleterious consequences on the myocardium were attenuated [30-32]. Secondly, carvedilol increases renal blood flow [33] and decreases cardiac sympathetic drive to a greater extent than selective beta-adrenergic blockers [34]. Moreover, it appears to suppress aldosterone production [35]. All together, these mechanisms could diminish myocardial injury during compensation and contribute to prevent further damage and future cardiovascular events. Thirdly, the novel mechanism hypothesized by Fallick $\mathrm{C}$ et al, could by restoring systemic 
venous capacitance, contribute to prevent additional episodes of decompensation and myocardial injury [23]. In summary, the observed beneficial effects of cautious uptitration of carvedilol, in decompensated patients in sinus rhythm, are very likely due its unique pharmacological characteristic of $\alpha$ and $\beta$ blocker [36].

\section{Limitations}

Our study is a retrospective, opened label, nonrandomized clinical investigation with a small sample. Initially, we compared two opposite therapeutic strategies. However, in view of the very favourable changes, induced by carvedilol on heart rate, neurohormonal activation and non-sustained ventricular arrhythmias (20); the responsible investigators decided to assign most patients to this particular protocol. Although, the survival results may have been influenced by their decision, our findings are congruent with those reported in the literature. Furthermore, the already mentioned differences of carvedilol, with other beta-adrenergic blockers, could explain our findings.

\section{Conclusions}

In Summary, in this investigation we can conclude with this analysis that indicated, the medical treatment with Carvedilol was significantly associated to survival, only in those patients who were in sinus rthyms and cautious uptitration of carvedilol, is still decompensated with sinus rhythm, increases long term survival

\section{Disclosure Section}

All the authors do not have any possible conflicts of interest.

\section{Funding}

Supported in part by Consejo de Desarrollo Científico, Humanístico y Tecnológico (M-1048-13-07-A). Universidad de Los Andes. Mérida, Venezuela.

\section{References}

[1] Tsutsui M, Tsuchihashi M and Takeshita A. Mortality and Readmission of hospitalized Patients with Congestive Heart Failure and Preserved Versus Depressed Systolic Function. Am J Cardiol 2001; 88: 530-533.

[2] Feinglass J, Lee PI, Mehta S, Schmitt B, Lefevre F, Gheorghiade M Systolic function, readmission rates, and survival among consecutively hospitalized patients with congestive heart failure. Am Heart J 1997; 134: 728-736.

[3] Solomon SD, Dobson J, Pocock S, Skali H, McMurray JJ, Granger CB et al. Candesartan in Heart failure: Assessment of Reduction in Mortality and morbidity (CHARM) Investigators. Influence of nonfatal hospitalization for heart failure on subsequent mortality in patients with chronic heart failure. Circ 2007; 116: 1482-1487.
[4] Konishi M, Maejima Y, Inagaki H, Clinical characteristics of acute decompensated heart failure with rapid onset symptoms. J Card Fail 2009; 15: 300-304.

[5] Schiff GD, Fung S, Speroff T, Mcnutt RA. Decompensated heart failure: Symptoms, patterns of onset and contributing factors. Am J Med 2003; 114: 625-630.

[6] Drazner MH, Hellkamp AS, Leier CV, Shah MR, Miller LW, Russell SD et al. Value of clinician assessment of hemodynamics in advanced heart failure. Circ Heart Fail 2008; 1: 170-177.

[7] Nohria A, Tsang SW, Fang JC, Lewis EF, Jarcho JA, Mudge $\mathrm{GH}$ et al. Clinical assessment identifies hemodynamic profiles that predict outcomes in patients admitted with heart failure. J Am Coll Cardiol 2003; 41: 1797-1784.

[8] Tuy T, Peacock WF. Fluid overload assessment and management in heart failure patients. Semin Nephrol 2012; 32: $112-120$.

[9] Khoury AM, Davila DF, Bellabarba G, Donis JH, Torres A, Hernandez L et al. Acute effects of digitalis and enalapril on the neurohormonal profile of chagasic patients with severe congestive heart failure. Int J Cardiol 1996; 57: 21-29.

[10] Gheorghiade M, Pang PS, Ambrosy AP, Lan G, Schmidt P, Filippatos G, Konstam M et al. A comprehensive, longitudinal description of the in-hospital and post-discharge clinical, laboratory, and neurohormonal course of patients with heart failure who die or are re-hospitalized within 90 days: analysis from the EVEREST trial. Heart Fail Rev 2012; 17: 485-509.

[11] Felker GM, Hasselblad V, Wilson Tang WH et al, Troponin I in acute decompensated heart failure: insights from the ASCEND-HF study. European Journal of Heart Failure 2012; 14: $1257-1264$.

[12] Peacock WF, Costanzo MR, De Marco T, Lopatin M, Wynne J, Mills RM et al. ADHERE Scientific Advisory Committee and Investigators. Impact of intravenous loop diuretics on outcomes of patients hospitalized with acute decompensated heart failure: insights from the ADHERE registry. Cardiology 2009; 113: 12-19.

[13] Vaz Pérez A, Otawa K, Zimmermann AV, Stockburger M, MüllerWerdan $U$ et al. The impact of impaired renal function on mortality in patients with acutely decompensated chronic heart failure. Eur J Heart Fail. 2010; 12: 122-128.

[14] Felker GM, Lee KL, Bull DA, Redfield MM, Stevenson LW, Goldsmith SR et al. NHLBI Heart Failure Clinical Research Network. Diuretic strategies in patients with acute decompensated heart failure. N Engl J Med. 2011; 364: 797-805.

[15] Konish M, Haraguchi G, Ohigashi H et al. Progression of Hyponatremia is Associated with Increased Cardiac Mortality in Patients Hospitalized for Acute Decompensated Heart Failure. J Cardiac Fail 2012; 18: 620-625.

[16] Mc Murray JJ. Clinical Practive. Systolic Heart Failure. N Eng. J Med 2010; 362: 228-38.

[17] McMurray JJ, Adamopoulos S, Anker SD, Auricchio A, Böhm M, Dickstein K et al. ESC Guidelines for the diagnosis and treatment of acute and chronic heart failure 2012. The Task Force for the Diagnosis and Treatment of Acute and Chronic Heart Failure 2012 of the European Society of Cardiology. Developed in collaboration with the Heart Failure Association (HFA) of the ESC. Eur J Heart Fail 2012; 14: 802-869. 
[18] Fonarow GC, Abraham WT, Albert NM, Stough WG, Gheorghiade M, Young JB et al. OPTIMIZE-HF Investigators and Coordinators, Influence of beta-blocker continuation or withdrawal on outcomes in patients hospitalized with heart failure: findings from the OPTIMIZEHF program. J Am Coll Cardiol. 2008; 52: 190-199.

[19] Orso F, Baldasseroni S, Fabbri G, Gonzini L, Lucci D, D'Ambrosi $\mathrm{C}$ et al. Italian Survey on Acute Heart Failure Investigators. Role of Beta Blockers in patients admitted for worsening heart failure in a real world: data from the Italian Survey on Acute Heart Failure. Eur J Heart Fail 2009; 11: 77-84.

[20] Lobo-Vielma L, Comenares-Mendoza H, Donis JH, Sanchez F, Perez A, Dávila DF. Acutely decompensated heart failure: Effects of carvedilol on clinical status, neurohormonal activation and ventricular arrhythmias. Int J Cardiol 2010; 144: 302-303.

[21] OH JK, Park SJ, Nagueh SF. Stablished and novel clinical applications of diastolic function assessment by echocardiography. Circ Cardiovasc Imaging 2011; 4: 444-455.

[22] Cotter G, Felker GM, Adams KF, Milo-Cotter O, O'Connor CM. The pathophysiology of acute heart failure--is it all about fluid accumulation? Am Heart J. 2008; 155: 9-18.

[23] Fallick C, Sobotka PA, Dunlap ME. Sympathetically mediated changes in capacitance: redistribution of the venous reservoir as a cause of decompensation. Circ Heart Fail. 2011; 4: 669-75.

[24] Gelman S, Mushlin PS. Catecholamine-induced changes in the splanchnic circulation affecting systemic hemodynamics. Anesthesiology 2004; 100: 434-439.

[25] Francis GS, Siegel RM, Goldsmith SR, Olivari MT, Levine TB, Cohn JN. Acute vasoconstrictor response to intravenous furosemide in patients with chronic congestive heart failure. Activation of the neurohumoral axis. Ann Intern Med. 1985, 103: 1-6.

[26] Waagstein F, Hjalmarson A, Varnauskas E, Wallentin I. Effect of chronic beta-adrenergic receptor blockade in congestive cardiomyopathy. Br Heart J. 1975; 37: 1022-1036.

[27] Packer M, Bristow MR, Cohn JN, Colucci WS, Fowler MB, Gilbert EM et al. The effect of carvedilol on morbidity and mortality in patients with chronic heart failure. U.S. Carvedilol Heart Failure Study Group. N Engl J Med. 1996; 334: 1349-1355.
[28] Metra M, Torp-Pedersen C, Cleland JG, Di Lenarda A, Komajda M, Remme WJ et al. COMET investigators. Should beta-blocker therapy be reduced or withdrawn after an episode of decompensated heart failure? Results from COMET. Eur J Heart Fail. 2007; 9: 901-909.

[29] Jondeau G, Neuder Y, Eicher JC, Jourdain P, Fauveau E, Galinier $\mathrm{M}$ et al. B-CONVINCED Investigators. B-CONVINCED: Beta-blocker CONtinuation Vs. INterruption in patients with Congestive heart failure hospitalizED for a decompensation episode. Eur Heart J. 2009; 30: 2186-2192.

[30] Kaye DM, Lefkovits J, Jennings GL, Bergin P, Broughton A, Esler MD. Adverse consequences of high sympathetic nervous activity in the failing human heart. J Am Coll Cardiol. 1995; 26: 1257-1263.

[31] Dávila DF, Núñez TJ, Odreman R, de Dávila CA. Mechanisms of neurohormonal activation in chronic congestive heart failure: pathophysiology and therapeutic implications. Int $\mathrm{J}$ Cardiol. 2005; 101: 343-346.

[32] Davila DF, Donis JH, Bellabarba G, Torres A, Casado J, Mazzei de Davila C. Cardiac afferents and neurohormonal activation in congestive heart failure. Med Hypotheses. 2000; 54: 242-253.

[33] Nikolaidis LA, Poornima I, Parikh P, Magovern M, Shen YT, Shannon RP. The effects of combined versus selective adrenergic blockade on left ventricular and systemic hemodynamics, myocardial substrate preference, and regional perfusion in conscious dogs with dilated cardiomyopathy. J Am Coll Cardiol. 2006; 47: 1871-1881.

[34] Azevedo ER, Kubo T, Mak S, Al-Hesayen A, Schofield A, Allan R et al. Nonselective versus selective beta-adrenergic receptor blockade in congestive heart failure: differential effects on sympathetic activity. Circulation 2001; 104: 2194-2199.

[35] Aggarwal A, Wong J, Campbell DJ. Carvedilol reduces aldosterone release in systolic heart failure. Heart Lung Circ. 2006; 15: 306-309.

[36] Hawkins NM, Petrie MC, Macdonald MR, Jhund PS, Fabbri LM, Wikstrand $\mathrm{J}$ et al. Heart failure and chronic obstructive pulmonary disease the quandary of Beta-blockers and Beta-agonists. J Am Coll Cardiol 2011; 57: 2127-2138. 